\title{
Bacteriological and Molecular Study of Klebsiella Pneumoniae Isolated from Patients with Urinary Tract Infections from Several Hospitals in Baghdad
}

\author{
Rasha Zaid Tariq Ahmed ${ }^{1}$, Teeba Fares Hadi ${ }^{2}$, Rana Mujahid Abdullah ${ }^{3}$ \\ ${ }^{1}$ Assist. Lecturer, Second Al-Karkh Education Directorate, Ministry of Education, Baghdad, Iraq, ${ }^{2}$ Assist. Lecturer, \\ AL-Rasheed University College Department of Biology, ${ }^{3}$ Prof., Department of Biology, College of Education Ibn-Al \\ Haitham, University of Baghdad, Baghdad, Iraq
}

\begin{abstract}
This study obtained 20 isolates of Klebsiella pneumoniae from out of 50 isolates collected from patients with urinary tract infection; all isoates were diagnosed and then the sensitivity test for isolates was performed using the discs method (Kirby-Bauer) to determine the resistance of K.pneumoniae to 10 antibiotics. The results detected that the highest resistance was against Ampicillin, Cefotaxim, and Piperacillin by $100 \%$ for each of them, while the rest of the antibiotics had a resistance percentage of $80 \%$ for Trimethoprim, $54 \%$ for Gentamicin and $65 \%$ for Azithromicin, however the lowest resistance was found against Imipenem, Chloramphenicol and Ofloxacin by $4 \%, 12 . \%$ and $35 \%$ respectively. The genomic bacterial DNA of studied isolates was extracted and the genotyped was performed identify the genetic relationships between the bacterial isolates using ERIC-PCR method. The results of the study showed the presence of 12 genotypes and also the results revealed the presence of two clones, each of which contains similar genotypes, while the rest of the isolates contain different genotypes.
\end{abstract}

Keywords: Klibsiella pneumoniae, antibiotic resistance, ERIC-PCR.

\section{Introduction}

Klebsiella pneumoniae is one of the most important genera of the Enterobacteraceae family. It is characterized by being Gram-negative bacilli, nonmotile, and lactose fermented, non spore forming, appear under the microscope are made up of thick edges, as the edges are curved outward and have rounded ends and have a capsule that increases its pathogenicity (Braun et al., 2004; Bensen 2001; Sambrook et al., 2001). It was called by this name in the nineteenth century in relative to its discoverer Edwin Klebs, a German-born scientist, in 1834 (Brise et al. 2005).

K.pneumoniae is one of the causative agents of opportunistic diseases in humans, including respiratory tract infection, Burns inflammation, wounds inflammation, septicemia, diarrhea, and liver abcesses (Zhang et al., 2018) .

Urine tract infection is one of the most common diseases caused by K. pneumoniae after Escherichia coli.
Moreover, it is become more dangerous for people who suffer from Diabetics, alcoholics, pulmonary deficiency, immune suppressive, and hospitalized patients especially in intensive care units, thus the accurate and rapid diagnosis is required to prevent the infection in hospitals (Li et al, 2012; Chiu et al., 2013; Guo et al. 2016).

K. pneumoniae bacteria possess many virulence factors including production of polysaccharide, capsule, serum resistance, production of iron siderophore, production of enterotoxin and urea and thus this bacteria with high virulent in addition to developing its resistance to many antibiotics (Navan-venzia et al., 2017; Dubey et al., 2013).

The major reasons for $K$. pneumoniae resistance to antibiotics including beta-lactam antibiotics is due to producing the broad-spectrum of beta-lactamase (ESBL) which is one of the most important problems of increased infection in hospitals, or through the changing of the permeability barrier or in the target site 
represented by penicillin binding protein, or alteration of outer membrane protein (Aghamohammad et al., 2018). Additionally, K. pneumoniae havine many Efflux Pumps that expel the antibiotic to the outside. Beta-lactam antibiotics include a group of antibiotics including penicillins, cephalosporins, carbenems and monobactam (Livermore, 2012; Sachse et al., 2012, Levinson., 2016).

Trimethoprim inhibits dihydro pterotese Synthetase (DHPS) and dihydro folate reductase (DHFR), which are involved in the synthesis of bacterial DNA, and $K$. pneumoniae showed high resistance to Trimethoprim. The reason for this resistance is via modulating the target site that bind with the enzymes, and also having encoded genes carrying on conjugate plasmids that may be acquired or given to other bacteria present in the same culture medium, as well as containing a flow mechanism that changes the membrane permeability (Shin et al., 2015).

Moreover, K. pneumoniae possesses multiple mechanisms to resist aminoglycoside; these mechanisms including its production of the three modulating enzymes: Acetyl transferase, Phospho transferase and Adenal transferase (Serio et al., 2017). Additionally, K. pneumoniae have several mechanisms of resistance to antibiotics including Quinolones antibiotics such as Ciprofloxacin, Norofloxacin, Levofloxacin and Ofloxacin. These mechanisms invovlved the modulation of the target site that bind with the antibiotics by triggering chromosomal genetic mutations in the genes encoding for DNA grease or Topoisase resulting in producton of different enzyme that is resistant to these antibiotics. K. pneumoniae also have OqxAB and QepA efflux Pumps on the outer membrane of the bacteria that serve to expel the antibiotic to the outside and prevent the permeability of it (Jacoby, 2017).

Genotyping method are important in finding the genetic affinity between bacterila isolates and also in the classification of bacteria, identification the sources of infection and characterization of the most pathogenic strain. There are several method of genotyping including Enterobacterial Repetitive Intergenic consensus method which is easy and rapid method that does not need long time and less expensive in comparison with other method (Goudarzi et al., 2011).

\section{Aims of the study:}

1. Isolation and diagnosis of Klebsiella pneumoniae from patients with urinary tract infections.
2. Determination the resistance and sensitivity of Klebsiella pneumonia to antibiotics.

3. Detection the genotyping and genetic relationships of Klebsiella pneumoniae using ERIC-PCR method.

\section{Materials and Method}

1. Bacteria isolation: 50 samples were collected from patients with urinary tract infections, male and female, from several hospitals in Baghdad (Central Child Hospital, Medical City, Al-Yarmouk Hospital, Child Protection Hospital) for the period from $1 / 10 / 2019$ to $6 / 12 / 2019$.

2. Diagnosis of Isolates: Samples were diagnosed using blood agar and MacConkey agar culture media. For final diagnosis of isolates, Vitek 2 system was used.

3. The sensitivity of the bacterial isolates was perfomed using the discs diffusion method (KirbyBauer) to determine the resistance of $K$. pneumoniae for 10 antibiotics included Ampicilin, Cefotaxime, Piperacillin, Gentamycin Sulfamethoxazole + Trimethoprime, Imipenem, Ciprofloxacin, Azithromycin, Ofloxacin and Chloramphenicol. The measurement of the inhibition zone diameter with around the antibiotic discs was perfomed and compared with the tables of international measurements (CLSI, 2017).

4. DNA Extraction: Genomic DNA extraction and Purification Kit was used to extract the bacterial DNA according to manufacturer instructions (Promega,USA).

5. The genotyping of K.pneumoniae: it was perfomed using PCR technique to detect the genotype of ERIC gene using the following primers:

\section{ERIC (F): $5^{\prime}$ - ATG TAA GCT CCT GGG GAT TCA C-3 \\ ERIC (R): $5^{\prime}$ - AAG TAA GTG ACT GGG GTG AGC G-3}

The results of this test included the production of bands with variable size. The reaction mixture included $20 \mu \mathrm{L}$ of GO Taq Green Master Mix 10, $2 \mu \mathrm{L}$ of DNA template, $2 \mu \mathrm{L}$ of each primer and $6 \mu \mathrm{L}$ of deionized (molecular) distilled water. The reaction conditions was programed according to the manufacturer instructions (Promga, USA) with some modifications as following (Mehr et al., 2017): 
1. The initial denaturation with the single cycle at $94^{\circ}$ C for 3-minute.

2. DNA amplification using one cycle at $94^{\circ} \mathrm{C}$. In order to attach the primer with DNA templet, 35 cycles was used and each cycle included the following steps:

A- Anneling stage : one cycle at $48^{\circ} \mathrm{C}$ for 1 minute.

B- Extension stage: at $72^{\circ} \mathrm{C}$ for 2 minutes.

C- Final Extension stage: one cycle at $72^{\circ} \mathrm{C}$ for 5 minutes.

6. The reaction products were separated using agarose gel (Bio Basic INC, Canada) (2\%) containing $5 \mu \mathrm{l}$ of Eithidium bromide (Promega USA), and using DNA ladder (100-1500) base pair with a voltage difference of 100 volts for 80 minutes and imaged using UV light (Optima, Japan).

\section{Results and Discussion}

After performing the laboratory tests, 20 isolates of Klibsiella pneumoniae were obtained from a total of 50 samples collected from patients with urinary tract infections from several hospitals in Baghdad.

K.pneumoniae are among the most important causes of urinary tract infections, fees, burn, respiratory tract infections and bacteremia (Le et al., 2012).

The results of this study revealed that the highest resistance and sensitivity of $K$. pneumoniae to the studied antibiotics was against Ampicillin by $100 \%$, Cefotaxime by $100 \%$, Piperacillin by $100 \%$ and for Trimethoprim by $80 \%$, while the rest of the antibiotics, the resistance percentage was $54 \%$ for Gentamicin and $65 \%$ for Azithromicin. Furthermore, the lowest resistance was against Imipenem 4\%, 12\% for Chloramphenicol and $35 \%$ for Ofloxacin as shown in Figure (1).

The findings of this study are in agreement with Mehr et al. (2017) study that determined the resistance of $K$. Pneumoniae isolates which isolated from several hospitals in Korea; it was found that the resistance rates to Imipenem, Ampicillin and Azithromicin were at rates of $(4 \%, 91 \%, 37 \%)$ respectively, as found in this study. Additionally, our results were consistent with Vasaikar et al., 2017 observation that found the resistance of $K$. pneumoniae isolates isolated from several hospitals in South Africa was $29.7 \%$ for Ciprofloxacin, $51 \%$ for Gentamicin and 70.8 for Trimethoprim.
Intereastingly, our results are consistent with the findings of Mehr et al. (2017) study where it showed resistance to Chloramphenicol by $14 \%$. The results of the current study also in agrrement with the results of the Algarawyi (2016) study that found the resistance to Piperacillin was $91.1 \%$; however it is inconsistent with the results of Vasaikar et al. (2017) study that reported the resistance to Piperacillin was $79 \%$. The results of this study also were consistent with the findings of Chasemian et al. (2018) study that isolated K. pneumonia which isolated from several hospitals in Tahran and found that the resitance to Cefotaxime was $94.6 \%$; although our results not consistent with the results of Babakhani et al., 2015 study where the resiatance to Ofloxacin was $86 \%$.

Moreover, the current results were also in agreement with the results of Mustafa (2018) study which showed that the resistance of $K$. pneumonia isolated from different clinical cases and from several hospitals in Baghdad were $32 \%$ and 84 against Ofloxacin Cefotaxim respectively. It is also consistent with Zedan-ALobadi study whose studied the resistance of $K$. pneumonia, isolated from several hospitals in Baghdad, against various antibiotics; it was revealed that the resistance rate was $2.5 \%$ for Imipenem, 95\% for Piperacillin, 97\% for Ampicillin, and 77.5 for Ttimethoprim.

The genetic relationship was determined by genotyping of the studied bacteria using the Enterobacterial Repetitive Intergenic Consensus (ERIC) method. The results of the current study showed the presence of a genetic relationship between $K$ pneumoniaea isolates and also the presence of 12 genotype; the molecular weight of these bundles ranged between (100-1000) base pair as shown in Table (1). Furthermore, the results as shown in Figure (3) found the presence of two clones, each of which contains similar genotypes and found between them a genetic affinity, while the rest of the isolates contain different genotypes. The first clone contained three isolates $(3,5,8)$ isolated from hospitalized patients in Al-Yarmouk Teaching Hospital in Baghdad, and from the patients with urinary tract infection. Additionally, these isolates also showed high resistance to antibiotics including Ampicilin, Cefotaxime, and Piperacilli. While the second clone contained four isolates $(8,9,5$ and 6$)$ and these isolates were with a genetic affinity.

Moreover, these isolated showed high resistance to antibiotics and showed sensitivity to Imipenem. Thus, the genotyping method is important in the field of finding 
the genetic relationship, determining its resistance and sensitivity to antibiotics, and determining the most pathogenic strain. The results of this study is in agreement with the findings of Mehr et al. (2017) which found that there are 12 genotype of $K$. pneumoniae isolates using ERIC-PCR method. While it is inconsistent with the findings of Wasfi et al. (2016) study which detected 21 genotype of $K$. pneumoniae using ERIC-PCR method.

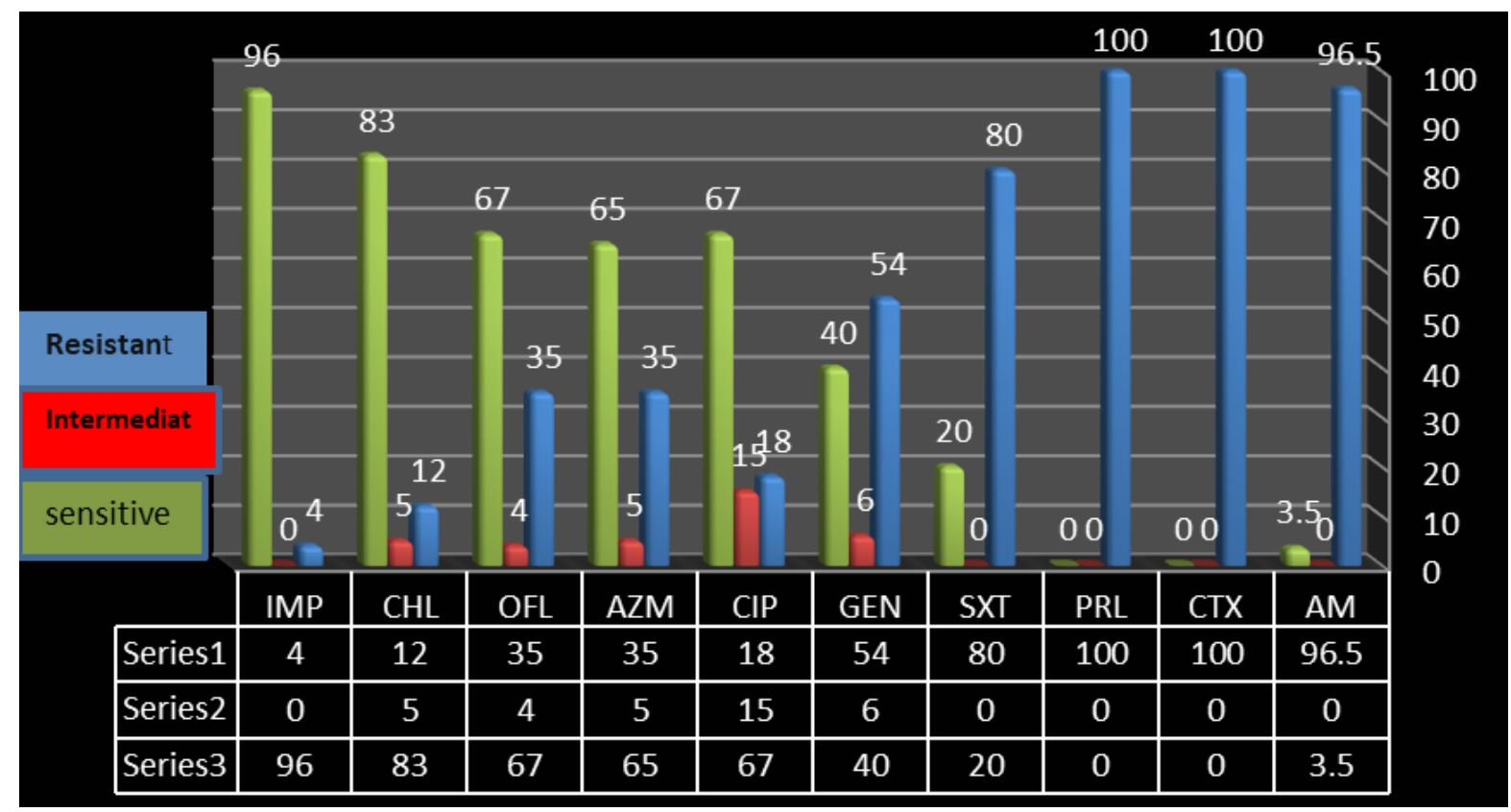

Figure (1): The resistance of Klibsiella pneumoniaea to various antibiotics

Ampicilin (AM), Cefotaxime (CTX), Piperacillin (PRL), Sulfamethoxazole + Trimethoprime (SXT), Gentamycin (GEN), Ciprofloxacin (CIP), Azithromycin (AZM), Ofloxacin(OFX), Chloramphenicol (CHL), Imipenem( IMP).

\section{12234567891011121314151617181920}

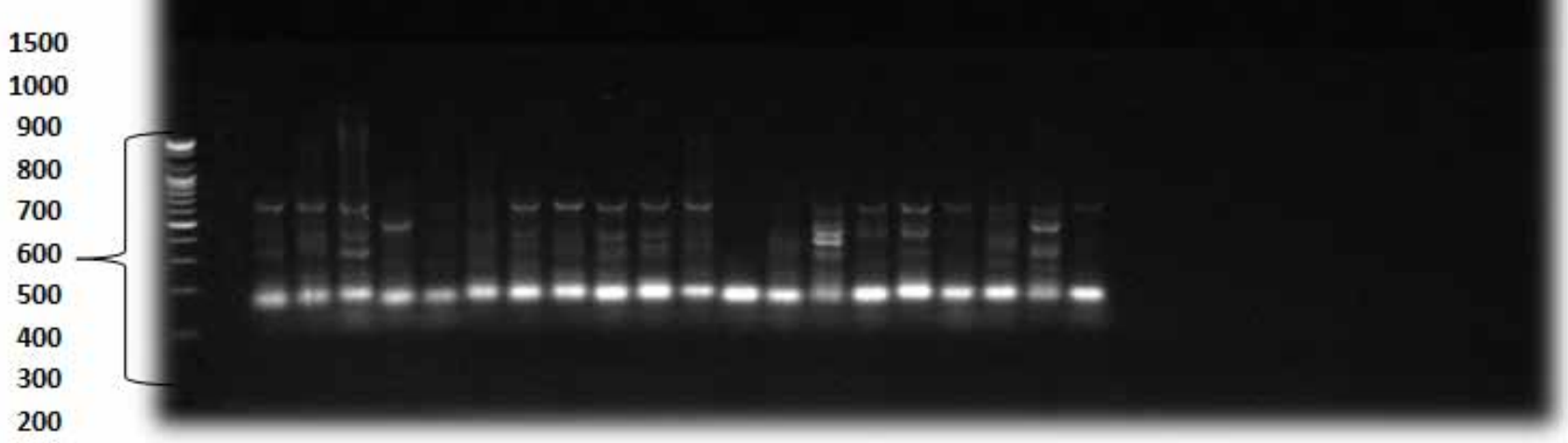

100

Figure (2): Electrophoresis of PCR product of isolates of $k$. pneumoniae using a specific primers for ERIC genotyping (100-1500 base pairs) on agarose gel (2\%) and with voltage difference of $100 \mathrm{~V}$ for 80 minutes. 
Table (1): The Molecular weights and percentages of bundles as products for ERIC-PCR method

\begin{tabular}{|c|c|c|c|}
\hline Bundles & The molecular weight (bp) & No. of isolates & Percentage (\%) \\
\hline ERIC1 & 100 & 1 & 5 \\
\hline ERIC2 & 200 & 8 & 40 \\
\hline ERIC3 & 250 & 2 & 70 \\
\hline ERIC4 & 300 & 8 & 45 \\
\hline ERIC5 & 350 & 5 & 25 \\
\hline ERIC6 & 400 & 3 & 15 \\
\hline ERIC7 & 500 & 13 & 65 \\
\hline ERIC8 & 600 & 2 & 10 \\
\hline ERIC9 & 700 & 1 & 5 \\
\hline ERIC10 & 800 & 1 & 5 \\
\hline ERIC11 & 900 & 2 & 10 \\
\hline ERIC12 & 1000 & 70 \\
\hline
\end{tabular}

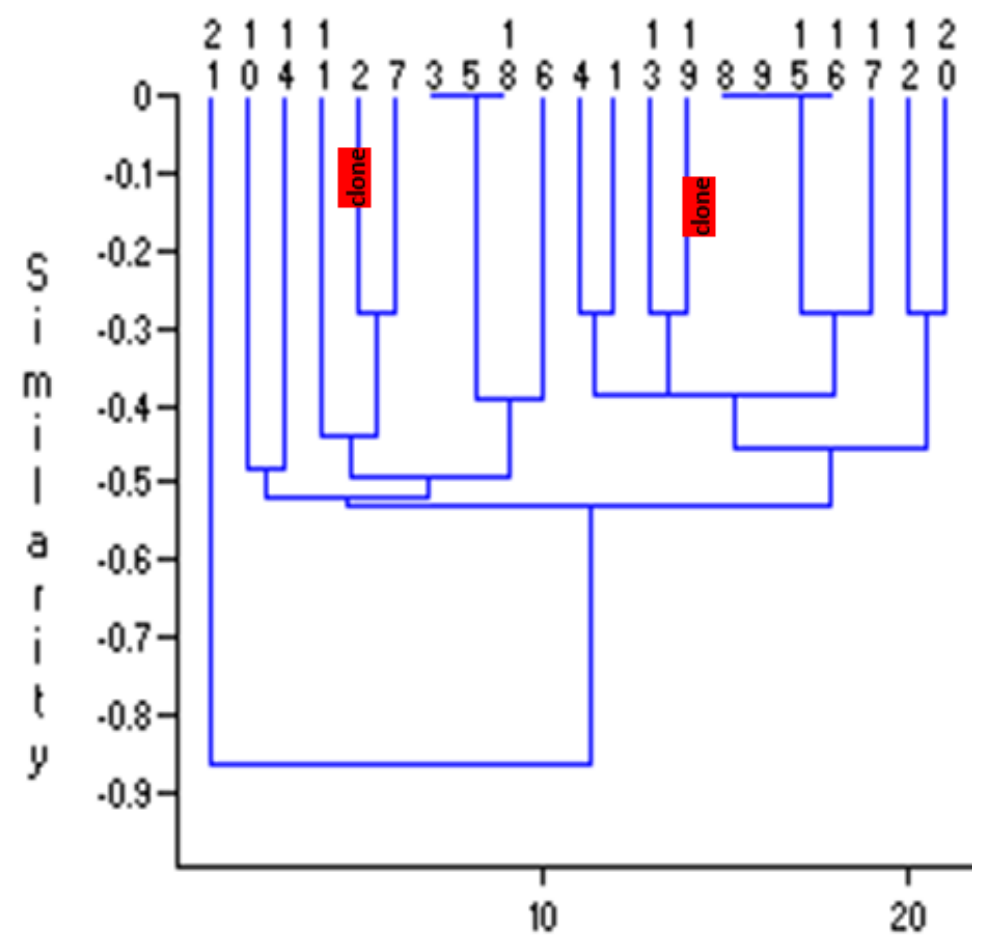

Figure (3): Dendogram of K. pneumonia isolates using Past Jaccard/up GMA program.

Financial Disclosure: There is no financial disclosure.

Conflict of Interest: None to declare.

Ethical Clearance: All experimental protocols were approved under the Ministry of Education and all experiments were carried out in accordance with approved guidelines.

\section{References}

1. Aghamohammad, S; Badmasti, F; Solgi, H; Aminzadeh, Z; Khodabandelo, Z; Shahcheraghi, F.(2018). First Report of Extended-Spectrum Betalactamase-Producing Klebsiella pneumoniae among Fecal Carriage in Iran: High Diversity of Clonal Relatedness and Virulence Factor Profiles. Microb. Drug Resist. Oct 02. 
2. Babakhani1, S; Shokri, S; Baharvand, M. (2015) Antibiotic resistance pattern of Klebsiella pneumonia isolated from nosocomial infections in Aleshtar hospital Lorestan province .Report of Health Care 1(2).

3. Benson, A. (2001). Microbiological Applications Laboratory in GeneralMicrobiology. (8th ed.). The McGraw-Hill Companies, U.S.A.

4. Braun, G. and Vidotto, M. (2004). Evaluation of Adherence, Hemagglutination, and Presence of Genes Codifying for Virulence Factors of Acinetobacter baumannii Causing Urinary Tract Infection. Mem Inst Oswaldo Cruz, Rio de Janeiro; 99(8): 839-844.

5. Brise, S.; Grimont F. and Grimont P. (2006). The Genus KlebsiellaIn: TheProkaryotes Proteobacteria, Dworkin, M. (ed.). (3rd ed.).Springer, New York, 159-19.

6. Chiu, S.; Wu, T.; Chuang, Y.; Lin, J.; Fung, C.; Lu, P.; Wang, J; Wang L.; Siu, K. and Yeh, K. (2013). National surveillance study on carbapenem Non-susceptible Klebsiella pneumoniae in taiwan: the emergence and rapid dissemination of kpc-2 carbapenemase. Plos One, 8(7): 1-7.

7. CLSI.(2017). Performance Standard for Antimicrobial Susceptibility Testing.27th ed. CSLI supplement M100.Wayne, PA:Clinical and Laboratory Standards Institute.32-41

8. Dubey, d.; Raza, F.; Sawhney, A. and Pandey, A. (2013). Klebsiella pneumoniae renal abscess syndrome: a rare case with metastatic of lungs, eye, and brain. Case Reports in Infect. Dis.: 1-3.., 12(373): 1-8.

9. Ghasemian, A; Shafiei, M; Eslami, M; Vafaei1, M; Nojoomi1, F; RajabiVardanjani, H .(2018) Molecular Typing of Klebsiella pneumoniae isolate using Repetitive Extragenic Palindromic SequenceBased PCR in a Hospital in Tehran, Iran. Enteric Pathogens.6(1):27-30.

10. Goudarzi, H.; Karimi, F.; Amoli, F. A.; Abedinyfar, Z.; Doustdar, F. and Mehrnejad, F. (2011). Genetic fingerprinting and antimicrobial susceptibility profiles of Pseudomonas aeruginosa isolates from eyeinfectins . Journal of clinical infection diseases, 6(1): 41-46.

11. Guo, Y.;Zhou, H.; Qin, L.; Pang, Z.; Qin, T.; Ren, H., et al. . (2016). Frequency, antimicrobial resistance and genetic diversity of Klebsiella pneumoniae in food samples. PLoS ONE 11:e0153561.

12. Jacoby, G.A.(2017).Plasmid-Mediated Quinolone Resistance. In: Mayers, DL.;Sobel, J.D.; Ouellette, M.; Kaye, K.S. and Marchaim, D .(eds) .Antimicrobil Drug Resistance. Mechanisms of Drug resistance (1). $2^{\text {nd }}$ ed .; Springer Switzerland. 265-268.

13. Lee, P.Y.;Costumbrado, J.;Hsu, C. and Kim, Y.H.(2012). Agarose Gel Electro phoresis for the Separation of DNA Fragments. J.Vis. Exp.62:5pp.

14. Levinson, W.(2016).Review of Medical Microbiology and Immunology $14^{\text {th }}$ ed.McGrawHill Higher Education.New York.821pp.

15. Li, J.; Sheng, Z.; Deng, M.; Bi1, S.; Hu, F.; Miao1, H.; Ji1, Z.; Sheng and Li, L. (2012). Epidemic of Klebsiella pneumoniae ST11 clone coproducing kpc-2 and 16S rRNA methylase rmtB in a chinese university hospital. BMC Infect. Dis

16. Livermore, D.M.(2012).Current epidemiology and growing resistance gram negative pathogens. Korean J. Intern. Med. 27(2):128-142.

17. Mehr, V, P; Shokoohizadeh, L, S; Mirzaee, M; Savari, M .(2017). Molecular Typing of Klebsiella pneumoniae Isolates by Enterobacterial Repetitive Intergenic Consensus (ERIC)-PCR. Infect Epidemio Microbiol .3(4):112-116.

18. Mustafa, MS.(2018).Molecular study of multidrug resistant Kelbsiella pneumoniae isolated from diffrented clinical samples. Msc Thesis. University of Baghdad. 143pp.

19. Navon-Venezia, S.;Kondratyeva, K. and Carattoli, A.(2017). Klebsiella pneumoniae: a major worldwide source and shuttle for antibiotic resistance FEMS Micrbiol. Rev. 41(3):252-275.

20. Sachse, S.; Bresan, S.; Erhard, M.; Edel, B.; Pfister, W.; Saupe, A. et al. .(2014). Comparison of multilocus sequence typing, RAPD, and MALDITOF mass spectrometry for typing of $\beta$-lactamresistant Klebsiella pneumoniae strains. Diagn. Microbiol. Infect. Dis. 80, 267-271.

21. Sambrook, J. and Rusell, D. W. (2001). Molecular cloning a laboratory manual. Cold spring Harbor, NY: cold spring Harbor Laboratory press.

22. Serio, A.W.; Magalhaes, M.L.; Blanchard, J.S. and Connolly, L.E.(2017) Drug Resistance. Mechanisms of Drug resistance. Volum1. $2^{\text {nd }}$ ed Spring Switzerland. 213-229. 
23. Shin, H.W.; Lim, J.; Kim, J.; Kown, G.C. and Koo, S.H.(2015 Characterization of TrimethoprimSulfamethoxazol resistance genes and their relatedness class 1 integron aninsertion sequence common region in gram negative bacilli .J. Microbiol. Biotechnol .25(1):137-142.

24. Vasaikar, S., Obi, L., Morobe, I., \& Bisi-Johnson, M. (2017). Molecular Characteristics and Antibiotic Resistance Profiles of Klebsiella Isolates in Mthatha, Eastern Cape Province, South Africa. International journal of microbiology, 8486742 .

25. Wasfi, R., Elkhatib, W. F., \& Ashour, H. M. (2016). Molecular typing and virulence analysis of multidrug resistant Klebsiella pneumoniae clinical isolates recovered from Egyptian hospitals. Scientific reports, 6 .

26. Zedan-Alobadi, TH. (2014). Molecular Identification of Klebsiell• pneumoniae Using Capsule Genes. Msc Thesis. Al-Nahrain University. $115 \mathrm{PP}$.

27. Zhang, S.; Yang, G.; Ye, Q.; Wu, Q.; Zhang, J and Huang, Y. (2018). Phenotypic and Genotypic Characterization of Klebsiella pneumoniae Isolated From Retail Foods in China. Frontiers in microbiology, 9, 289. 